\title{
Fractional wavelet transform using an unbalanced lifting structure
}

Y. Hakan Habiboğlu

Kivanc Kose

A. Enis Çetin 


\title{
Fractional Wavelet Transform Using an Unbalanced Lifting Structure
}

\author{
Y. Hakan Habiboğlu, Kivanc Kose and A. Enis Çetin \\ Department of Electrical and Electronics Engineering \\ Bilkent University \\ Bilkent, 06800, Ankara, Turkey
}

\begin{abstract}
In this article, we introduce the concept of fractional wavelet transform. Using a two-channel unbalanced lifting structure it is possible to decompose a given discrete-time signal $x[n]$ sampled with period $T$ into two sub-signals $x_{1}[n]$ and $x_{2}[n]$ whose average sampling periods are $p T$ and $q T$, respectively. Fractions $\mathrm{p}$ and $\mathrm{q}$ are rational numbers satisfying the condition: $1 / p+1 / q=1$. The low-band sub-signal $x_{1}[n]$ comes from $[0, \pi / p]$ band and the high-band wavelet signal $x_{2}[n]$ comes from $(\pi / p, \pi]$ band of the original signal $x[n]$. Filters used in the lifting structure are designed using the Lagrange interpolation formula. It is straightforward to extend the proposed fractional wavelet transform to two or higher dimensions in a separable or non separable manner.
\end{abstract}

Keywords: Lifting, wavelet transform, multirate signal processing

\section{INTRODUCTION}

Lifting structures provide computationally efficient implementation of the wavelet transform ${ }^{1-7}$. They found applications in image and video coding and signal and image analysis applications. ${ }^{8-10}$ Modified versions of the balanced wavelet structures were also proposed for fractional scalability of the images, and videos ${ }^{11}$. However, lifting structures providing scalable subsignals have not been developed.

In this article, new wavelet lifting structures producing unbalanced decomposition of the input signal are introduced. In standard lifting structures the input signal is first decomposed into even and odd indexed samples using the lazy wavelet transform as shown in Fig. 1. In the proposed unbalanced lifting structure, a structure similar to the lazy filterbank forwards every $p^{t h}$ sample of the original signal to the upper-branch and remaining $p-1$ samples out of $\mathrm{p}$ samples go to the lower branch. Discrete-time update and prediction filters interconnect the upper and lower branches, respectively. Discrete-time filters operating in the lower sampling rates are designed using the Lagrange interpolation formula which is also used in many filterbank designs including our filterbanks, Smith-Barnwell and Daubechies filterbanks. In this way, two sub-signals with different sampling rates are obtained from the original signal. When the input signal has a sampling rate of $\mathrm{T}$ the upper- and lower- branches of the unbalanced lifting filterbank have sampling rates of $p T$ and $T p /(p-1)$, respectively. In standard balanced lifting the sampling periods of upper and lower branches are the same: $2 T$.

The unbalanced lifting decomposition can be easily generalized to other sampling strategies in which the upper-branch has a sampling rate of $p T$ and the lower-branch has a sampling rate of $q T$ with the property that

$$
\frac{1}{p}+\frac{1}{q}=1
$$

Perfect reconstruction can be easily achieved by changing the signs of the filters in the reconstruction part of the ordinary balanced lifting structures.

In Section 2, an example filterbank design with $p=3: 1$ and $q=3: 2$ is presented. In Section 3, another filterbank example with $p=3: 2$ and $q=3: 1$ is presented. In Section 4, two-dimensional (2D) filterbank design examples are presented.

Further author information:

Y. Hakan Hakan Habiboğlu: E-mail: yhakan@ee.bilkent.edu.tr, Telephone: +90 3122901525

Kivanc Kose: E-mail: kkivanc@ee.bilkent.edu.tr, Telephone: +90 3122901164

A. Enis Çetin: E-mail:cetin@bilkent.edu.tr, Telephone: +90 3122901477

Independent Component Analyses, Wavelets, Neural Networks, Biosystems, and Nanoengineering IX, edited by Harold Szu, Liyi Dai, Proc. of SPIE Vol. 8058, 805805 · @ 2011 SPIE

CCC code: $0277-786 \mathrm{X} / 11 / \$ 18 \cdot$ doi: $10.1117 / 12.882408$

Proc. of SPIE Vol. 8058 805805-1 


\section{UNBALANCED LAZY FILTERBANK AND LIFTING STRUCTURES}

An unbalanced lazy filterbank for $p=3: 1$ and $q=3: 2$ is shown in Figure 2. In the upper-branch a regular downsampling block by a factor of three is used. In the lower-branch the downsampling block for $q=3: 2$ is used. In Figure 2, the signal $x[n]$ is fed to the lazy filterbank and outputs of downsampling blocks are shown. Every $3^{\text {rd }}$ sample of the original signal goes to the upper-branch and remaining samples appear in the lower branch.

We describe the update and the prediction filter design for the unbalanced lifting structure in the following two subsections.

\subsection{Update Filter}

We first review with the regular balanced lifting filterbank to illustrate the framework of the unbalanced lifting. Consider the lazy filterbank shown in Figure 1.

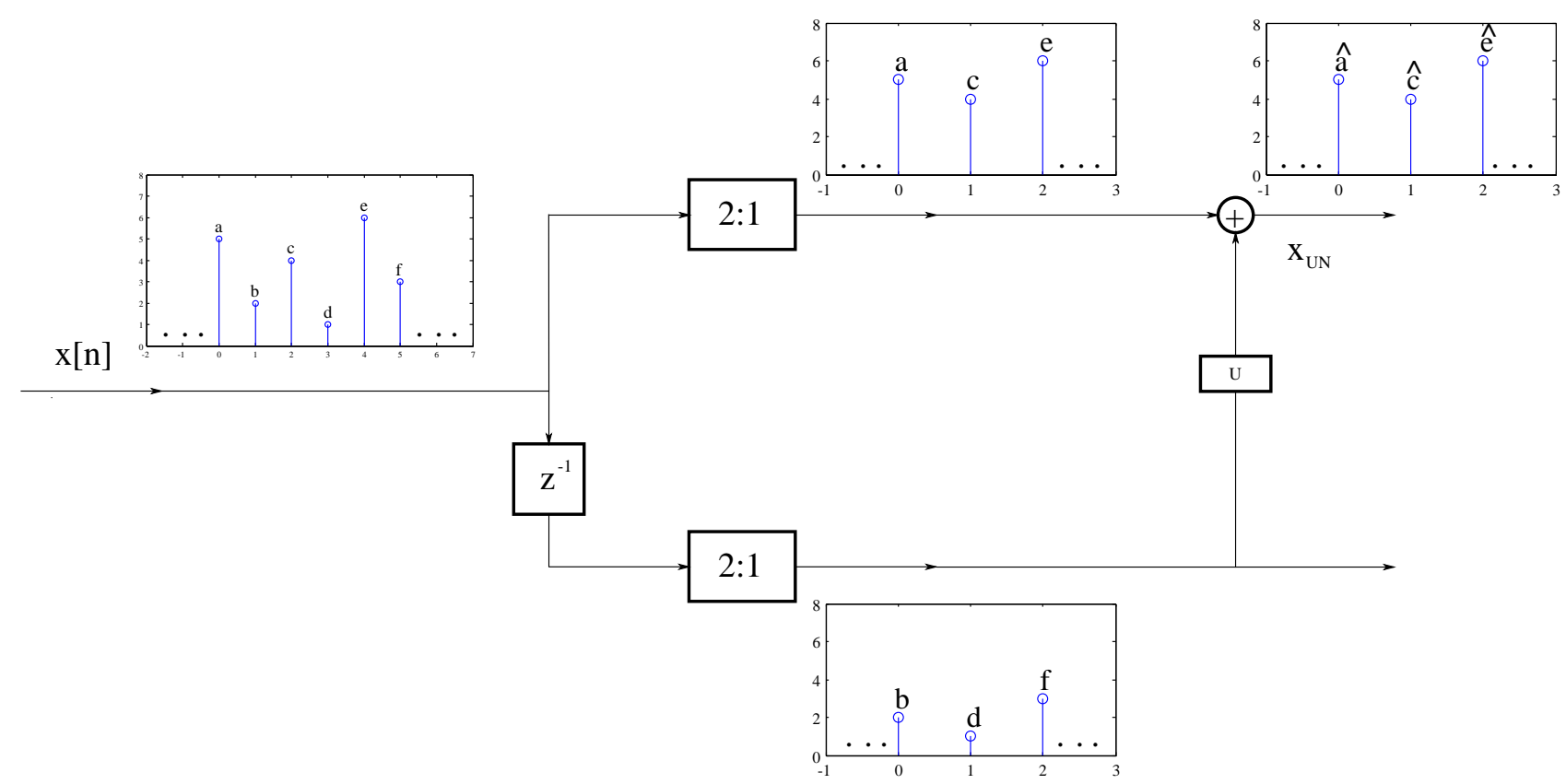

Figure 1. Lazy filterbank based regular lifting structure.

The signal $x[n]$ is divided into two sub-signals containing even and odd indexed samples of the original signal. The upper branch should be aliasing free as much as possible. The update filter tries to cancel aliasing in Figure 1. In many two-channel perfect reconstruction filter banks the Lagrange filter $h_{l}[n]=\left\{\frac{1}{4}, \frac{1}{2}, \frac{1}{4}\right\}$ is used for smoothing the signal ${ }^{4}$. For example, in Figure 1, the even indexed sample $c$ is linearly estimated using the odd indexed samples $b$ and $d$ by the update filter, and it is replaced by the value $\left(\frac{b+d}{2}+c\right) / 2$ or

$$
x_{u}[1]=\hat{c}=\frac{b}{4}+\frac{c}{2}+\frac{d}{4}
$$

after updating. All the other samples of the upper-branch sub-signal $x_{u}[n]$ are updated in a similar manner using the linear Lagrange filter.

The above update filter is based on linear interpolation. Other higher order Lagrange filters can be also used to construct the update filter $6,12,13$. For example, the Lagrange filter $h_{l}[n]=\left\{\frac{-1}{32}, 0, \frac{9}{32}, \frac{1}{2}, \frac{9}{32}, 0, \frac{-1}{32}\right\}$ based on higher order polynomial interpolation is widely used in filter bank design ${ }^{1-7,14-17}$. In Figure 1, this means that the sample $c$ is estimated by the update filter $c^{\prime}=\frac{9}{32}(b+d)-\frac{1}{32}(f+x[-1])$ and the corresponding upper branch signal is given by $\hat{c}=\frac{c+c^{\prime}}{2}$. 


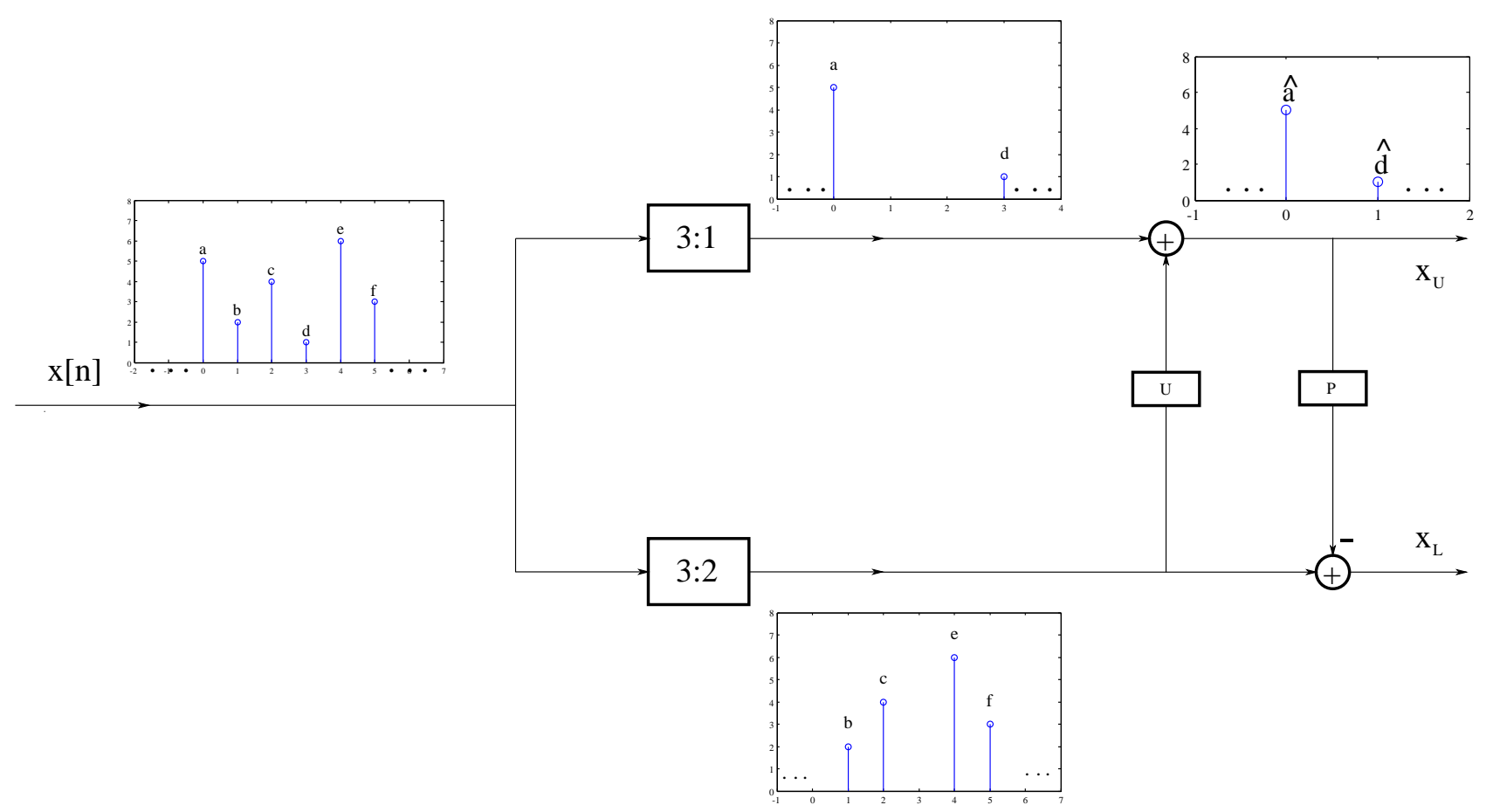

Figure 2. Unbalanced lifting scheme with $p=3: 1$ and $q=3: 2$ downsampling ratios.

In Figure 2 an unbalanced lifting structure is shown with downsampling ratios $3: 1$ and $3: 2$ in the upper and lower branches, respectively. Similar to the regular balanced filterbank case, the upper branch sample $d$ can be estimated using the neighboring lower branch samples $b, c, e$, and $f$ and an estimate of the sample $d$ is given as follows:

$$
d^{\prime}=\frac{b+2 c+2 e+f}{6},
$$

which is the output of the update filter linking the lower branch to the upper branch. Since samples $c$ and $e$ are closer to the sample $d$ compared to $f$ and $b$ more weight is given to the samples $c$ and $e$. The sample $d$ and the output of the filter is linearly combined and the updated sample is obtained as follows

$$
\hat{d}=\frac{2 d^{\prime}}{3}+\frac{d}{3}
$$

Since the downsampling is by a factor of three in the upper-branch original signal must be filtered with a low-pass filter with a cut-off frequency of $\pi / 3$. We could have used $\hat{d}=\frac{d^{\prime}}{2}+\frac{d}{2}$ but this does not produce as good frequency response as (4). Therefore, the upper branch output sample is given by

$$
\hat{d}=\frac{2 b}{18}+\frac{4 c}{18}+\frac{6 d}{18}+\frac{4 e}{18}+\frac{2 f}{18}
$$

The corresponding filter coefficients are given as follows

$$
h_{l}[n]=\left\{\frac{2}{18}, \frac{4}{18}, \frac{6}{18}, \frac{4}{18}, \frac{2}{18}\right\}
$$

The frequency response of this filter is plotted in Figure 3. As it can be observed from this figure the cut-off frequency of this filter is $\pi / 3$.

Other samples of the upper branch of the filterbank are smoothed in a similar manner. In Equation (5), each sample of the upper branch sub-signal is updated using four neighboring samples of the lower branch. It is possible to smooth the samples of the upper branch further by using more samples from the lower branch without 


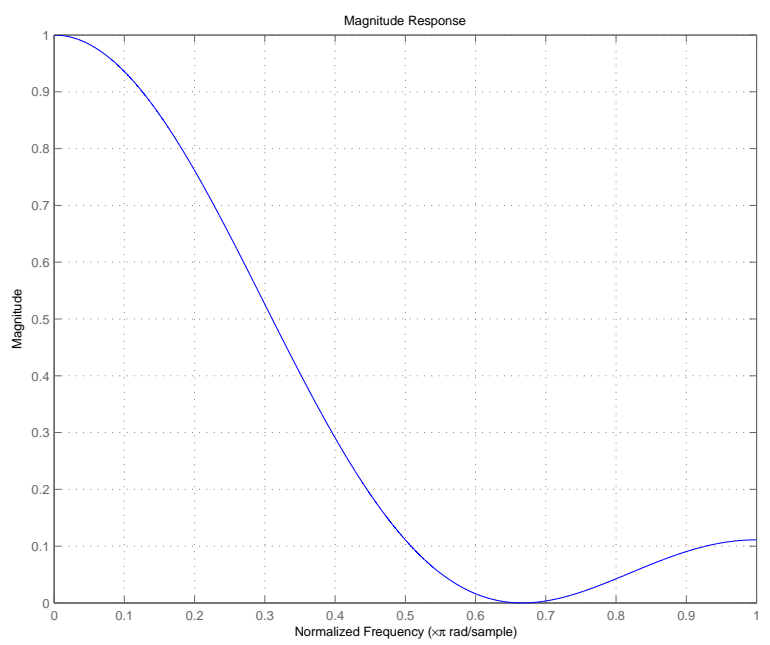

Figure 3. Frequency response of the filter in Equation (6).

effecting the perfect reconstruction capability of the filterbank. In this case, Lagrange interpolation formula or other interpolation methods can be used to determine the update filter coefficients. In general,

$$
x_{U}[n]=\frac{1}{9} x[3 n-2]+\frac{2}{9} x[3 n-1]+\frac{3}{9} x[3 n]+\frac{2}{9} x[3 n+1]+\frac{1}{9} x[3 n+2]
$$

where $x[n]$ is the original input signal to the filterbank.

\subsection{Prediction Filter}

Samples of the lower branch are estimated from the upper branch and difference is transmitted to the receiver. Lower branch samples can be predicted from the upper branch using the updated samples $\hat{a}, \hat{d}, \hat{g}, \ldots$ (see Fig.2). The prediction filter can be as simple as the identity operator selecting the nearest upper branch sample as an estimate of the lower branch sample:

$$
\begin{aligned}
& x_{L}[0]=b-\hat{a}, \\
& x_{L}[1]=c-\hat{d}, \\
& x_{L}[2]=e-\hat{d}, \\
& x_{L}[3]=f-\hat{g},
\end{aligned}
$$

Other samples of $x_{L}[n]$ can be determined in a similar manner. Although the above prediction strategy is very simple and computationally efficient, the above predictor is not a good estimator. we can use Lagrange interpolation and obtain:

$$
\begin{aligned}
& x_{L}[0]=b-(2 \hat{a}+\hat{d}) / 3 \\
& x_{L}[1]=c-(2 \hat{d}+\hat{a}) / 3 \\
& x_{L}[2]=e-(2 \hat{d}+\hat{g}) / 3 \\
& x_{L}[3]=f-(2 \hat{g}+\hat{d}) / 3
\end{aligned}
$$


In general,

$$
x_{L}(n)= \begin{cases}x\left[\frac{3 n+2}{2}\right]-\frac{2 x_{U}\left[\frac{n}{2}\right]+x_{U}\left[\frac{n}{2}+1\right]}{3}, & \mathrm{n} \text { is even } \\ x\left[\frac{3 n+1}{2}\right]-\frac{2 x_{U}\left[\frac{n+1}{2}\right]+x_{U}\left[\frac{n-1}{2}\right]}{3}, & \mathrm{n} \text { is odd }\end{cases}
$$

where $x[n]$ is the original input signal to the filterbank.

To determine $x_{L}[0]$ the sample $b$ is estimated using the two nearest upper branch samples $\hat{a}$ and $\hat{d}$. Let $P(t)$ be the Lagrange interpolator based on the samples $\hat{a}$ and $\hat{d}$ :

$$
P(t)=\hat{a} \ell_{o}(t)+\hat{d} \ell_{1}(t)
$$

where $\ell_{i}(t)$ are the Lagrange basis polynomials. The function $P(t)$ is constructed using the input data pairs $(\hat{a}, 0)$ and $(\hat{d}, 3 T)$. Since the sample $b$ occurs at $t=T, \ell_{o}(T)=2 / 3$ and $\ell_{1}(T)=1 / 3$. Therefore the Lagrange interpolation gives more weight to the sample $\hat{a}$ because it is nearer to the sample $b$ compared to $\hat{d}$ to determine $x_{L}[0]$ in (9). Similarly, more weight is given to the sample $\hat{d}$ because it is nearer to the sample $c$ compared to $\hat{a}$ to determine $x_{L}[1]$ etc. As it can be seen from the above equations the predictor is a time varying filter. The predictor can even be an adaptive LMS-type filter as described in references ${ }^{4,14,18}$. The adaptive prediction tries to remove as much information as possible from the lower-branch using $x_{L}[n]$ samples. In this case the computational complexity is higher than the predictors in (8) and (9). Other samples of $x_{U}[n]$ can be also used by the predictor filter. In this case higher order Lagrange interpolation formula needs to be used ${ }^{4,13}$.

It is trivial to design the reconstruction filterbank as in regular lifting structures. At the reconstruction stage signs of filters $U$ and $P$ are changed and sub-signal samples are realigned to obtain the original signal $x[n]$.

\section{UNBALANCED FILTERBANK WITH $p=3: 2$ AND $q=3: 1$}

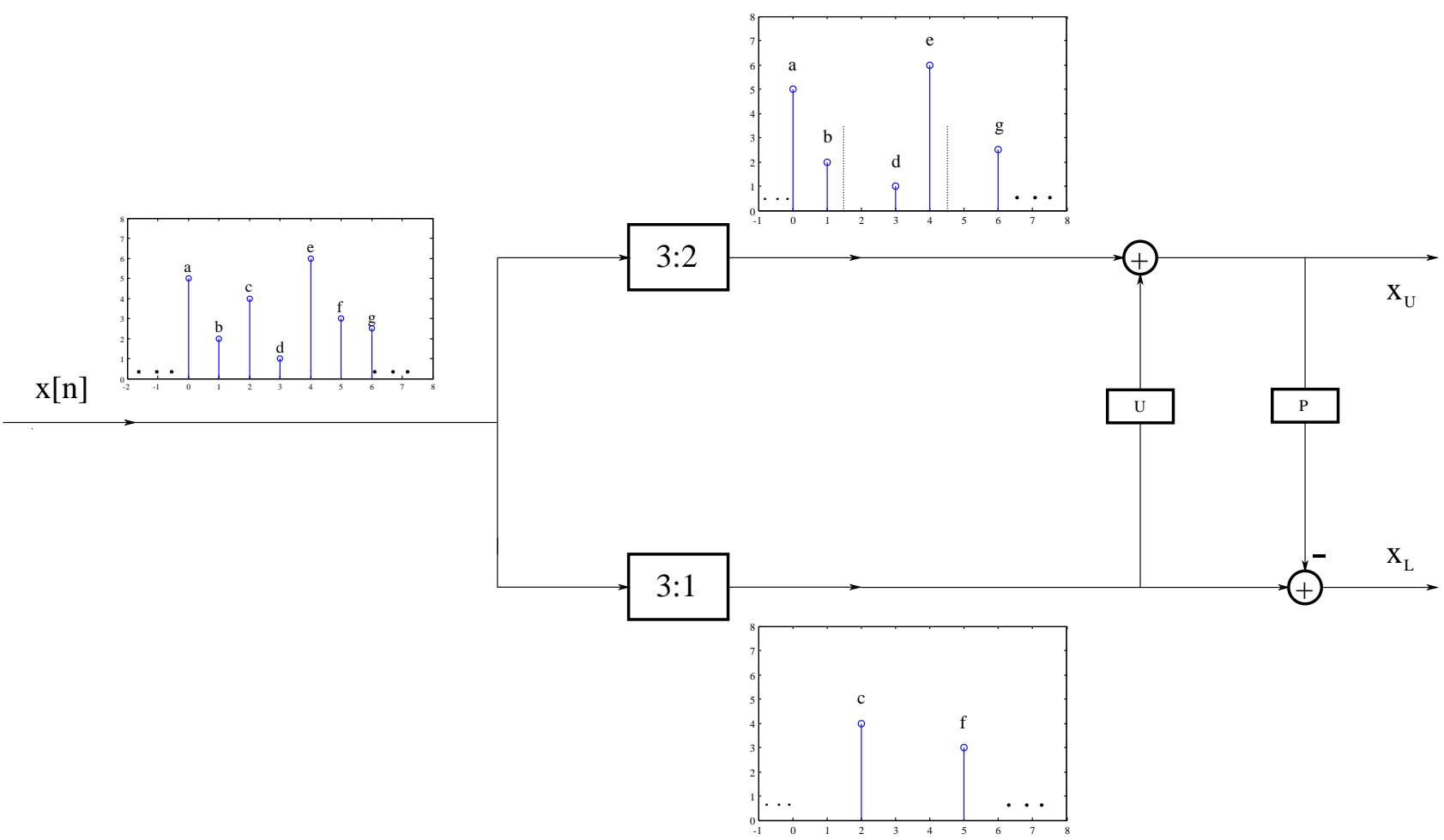

Figure 4. Unbalanced lifting structure with $\mathrm{p}=3: 2$ and $\mathrm{q}=3: 1$.

In Figure 4, the upper-branch has a downsampling ratio of $3: 2$ and the lower-breach has a downsampling ration of $3: 1$. In this case, samples of the upper-branch are downsampled in a non-uniform manner. In the 
upper branch the original shape of the signal has to be preserved as much as possible in practical applications including image and video processing. Therefore, the samples of the upper branch should be transformed to a uniformly sampled grid.

For example, in Figure 4, we need to estimate $x[1.5], x[4.5], \ldots$ using the lower-breach samples and the nearest current samples of the upper-branch, which are $x[1], x[4], \ldots$, respectively.

We can use linear interpolation and obtain the following updated values:

$$
x_{U}[1]=\hat{x}[1.5]=\frac{b+c}{2}, \quad x_{U}[3]=\hat{x}[4.5]=\frac{e+f}{2} \ldots
$$

One can also use other lower-branch samples as well during estimation without affecting perfect reconstruction.

Samples of $x_{U}[2 n]$ can be set as $x[3 n]$ or it is also possible to update other samples of the upper-breach using the lower branch:

$$
x_{U}[0]=a, \quad x_{U}[2]=\frac{1}{3} c+\frac{2}{3} d, x_{U}[4]=\frac{1}{3} f+\frac{2}{3} g, \ldots
$$

In general,

$$
x_{U}(n)= \begin{cases}\frac{x[1.5 n-1]+2 x[1.5 n]}{3}, & \mathrm{n} \text { is even and } \mathrm{n} \neq 0 \\ \frac{x[1.5(n-1)+1]+x[1.5(n+1)-1]}{2}, & \mathrm{n} \text { is odd }\end{cases}
$$

where $x[n]$ is the original input signal to the filterbank.

As usual, the prediction filter tries to remove as much information from the lower-breach as possible. For example, the samples $c$ can be predicted as follows, $x_{L}[1]=c-x_{U}[1]$, and, similarly $x_{L}[2]=f-x_{U}[3]$, etc...

Similar to the structure in Section 2, other samples of the upper-branch output $x_{U}[n]$ can be also used by a Lagrange interpolator for prediction purposes without effecting perfect reconstruction resulting a better quality sub-signal $x_{L}[n]$ as follows:

$$
x_{L}[1]=c-\left(\frac{2}{3} x_{U}[1]+\frac{1}{3} x_{U}[2]\right)
$$

where the predictor uses the same weights as in Equation (8) because the sample $x_{U}[1]$ occurs at $t=1.5 T$ and $x_{U}[2]$ occurs at $t=3 T$, and the sample $c$ occurs at $t=2 T$ as shown in Fig. 4 . As a result the Lagrange interpolator gives more weight to $x_{U}[1]$ compared to $x_{U}[2]$. Similarly, the next sample is predicted as follows

$$
x_{L}[2]=f-\left(\frac{2}{3} x_{U}[3]+\frac{1}{3} x_{U}[4]\right)
$$

In general,

$$
x_{L}[n]=x[3 n-1]-\left(\frac{2}{3} x_{U}[2 n-1]+\frac{1}{3} x_{U}[2 n]\right)
$$

where $x[n]$ is the original input signal to the filterbank.

It is straightforward to design the reconstruction filterbank as in regular lifting structures. At the reconstruction stage signs of filters $U$ and $P$ are changed and sub-signal samples are realigned to obtain the original signal $x[n]$.

\section{EXTENSION FOR TWO-DIMENSIONAL SIGNALS}

A N-dimensional filter $T\left(x_{1}, x_{2} \ldots x_{N}\right)$ is seperable if it can be represented as

$$
T\left(x_{1}, x_{2} \ldots x_{N}\right)=T_{1}\left(x_{1}\right) T_{2}\left(x_{2}\right) \ldots T_{N}\left(x_{N}\right)
$$

Assume that the two-dimensional (2D) filter $f(x, y)$ is seperable. Therefore it can be represented as follows

$$
T(x, y)=T_{1}(x) T_{2}(y)
$$


For a 2D signal or an image, Equation (19) corresponds to first filtering the signal in horizontal direction by $T_{1}(x)$ and then filtering the output signal in vertical direction by $T_{2}(y)$.

In Figure 5, the seperable filterbank structure for the unbalanced lifting wavelet transform with $p=3: 2$ and $q=3: 1$ is given. The input image is first downsampled in the horizontal direction. Then the update and prediction filters are applied to the downsampled images. The intermediate output signals $X_{U}$ and $X_{L}$ are then downsampled in the vertical direction and the update and prediction filters are applied again. As an example,

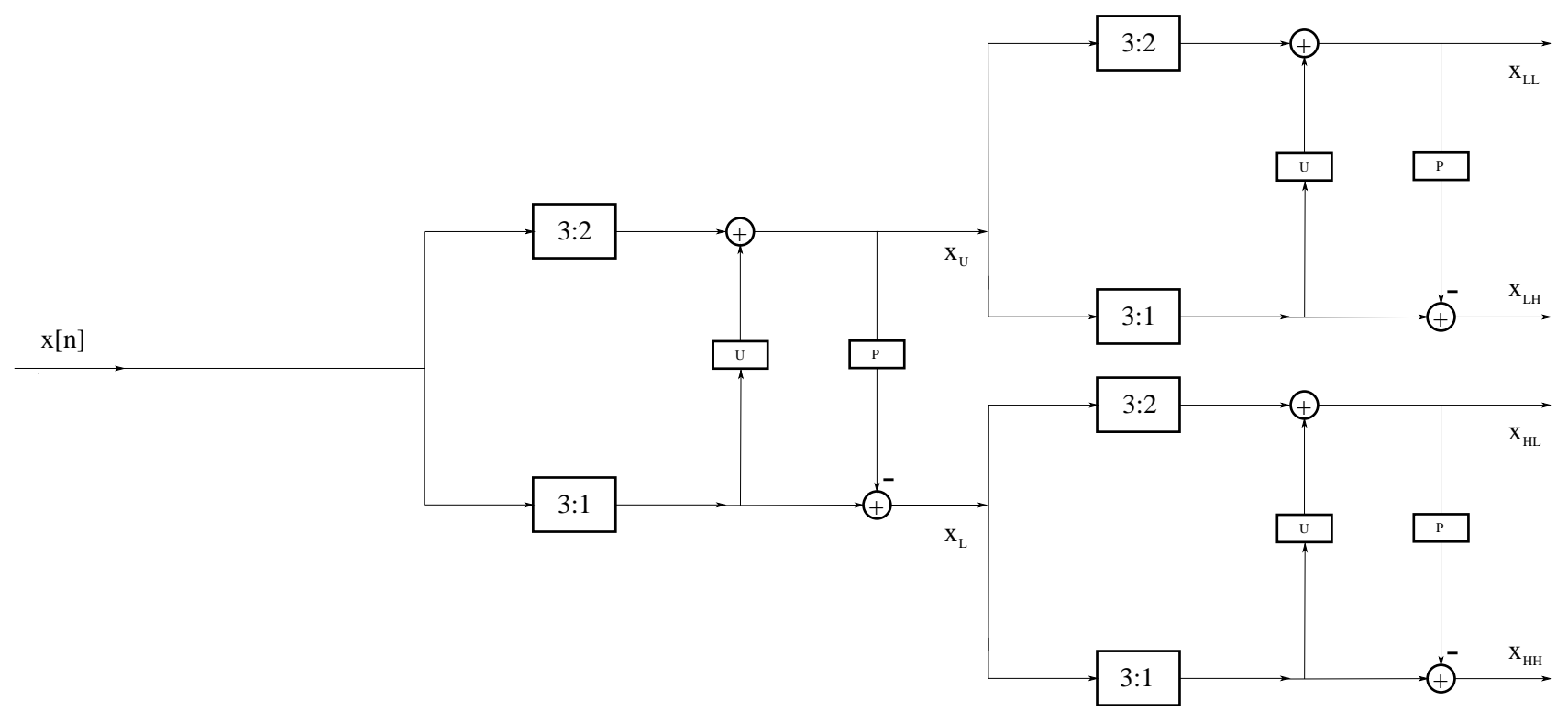

Figure 5. Extension of the unbalanced lifting structure with $\mathrm{p}=3: 2$ and $\mathrm{q}=3: 1$ to $2 \mathrm{D}$.

the image given at Figure 6 is fractional wavelet transformed using the scheme presented in Figure 5 . The transformed image is given at Figure 7. As another example, the image given at Figure 8 is wavelet transformed in Figure 2 using the fractional wavelet transform with $p=3: 1$ and $q=3: 2$ which is a 2D extended version of the filterbank described in Section 2.

It is also possible to develop non-seperable fractional filterbanks.

\section{CONCLUSION}

In this article, fractional wavelet transform (FWT) methods are developed using two-channel unbalanced lifting structures. Sub-signals have different average sampling rates.

The discrete time signal $x[n]$ sampled with period $T$ can be decomposed into two sub-signals with average sampling periods of $p T$ and $q T$, respectively. Example lifting structures for $p=3: 1$ and $q=3: 2$ and $p=3: 2$ and $q=3: 1$ cases are presented.

The FWT method can be easily extended to two or higher dimensions. Image transformation examples are presented.

\section{ACKNOWLEDGMENTS}

The research leading to there results has received funding from the European Community's Seventh Framework Programme (FP7-ENV-2009-1) under grant agreement no FP7-ENV-244088 "FIRESENSE - Fire Detection and Management through a Multi-Sensor Network for the Protection of Cultural Heritage Areas from the Risk of Fire and Extreme Weather". 


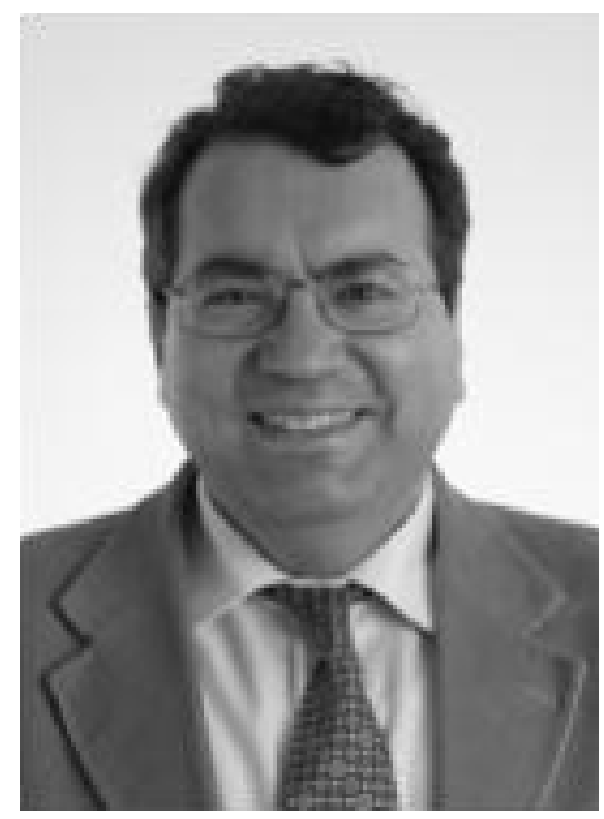

Figure 6. Example image.

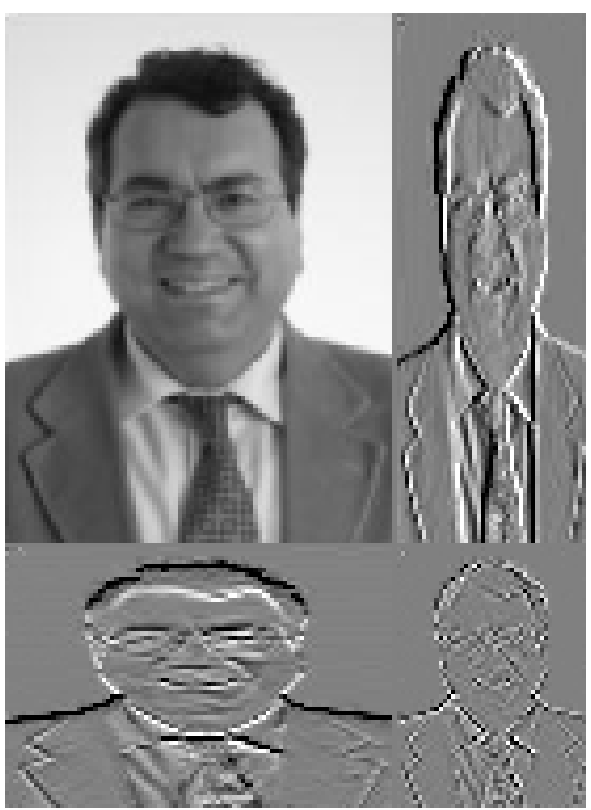

Figure 7. 2D unbalanced lifting wavelet transformed image with $\mathrm{p}=3: 2$ and $\mathrm{q}=3: 1$. Highband subimages are amplified by a factor of three.

\section{REFERENCES}

[1] Mallat, S., [A Wavelet Tour of Signal Processing, Second Edition (Wavelet Analysis \& Its Applications)], Academic Press, 2 ed. (Sept. 1999).

[2] Sweldens, W., "The lifting scheme: A custom-design construction of biorthogonal wavelets," Applied and Computational Harmonic Analysis 3(2), 186 - 200 (1996).

[3] Daubechies, I. and Sweldens, W., "Factoring wavelet transforms into lifting steps," Journal of Fourier Analysis and Applications 4, 247-269 (1998). 10.1007/BF02476026.

[4] Kim, C. W., Ansari, R., and Cetin, A. E., "A class of linear-phase regular biorthogonal wavelets," in 


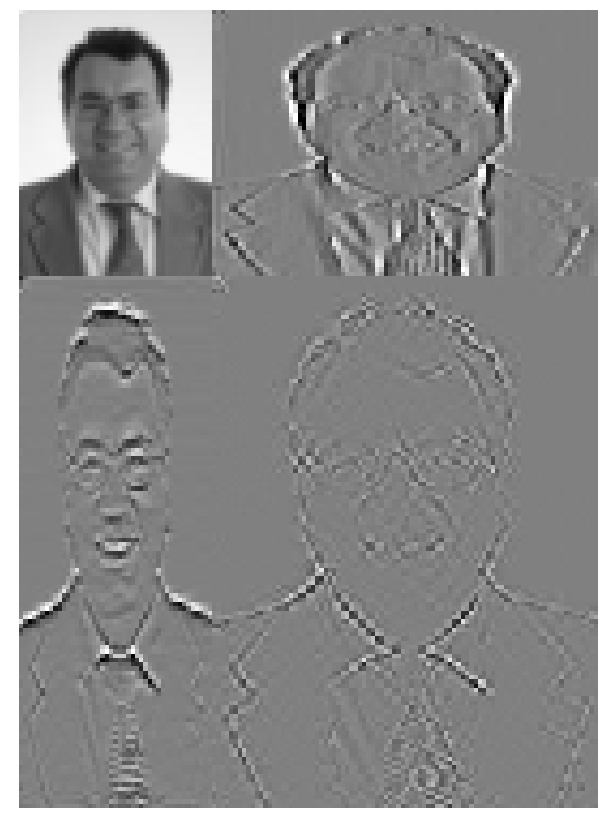

Figure 8. 2D unbalanced lifting wavelet transformed image with $p=3: 1$ and $q=3: 2$. Highband subimages are amplified by a factor of three.

[Acoustics, Speech, and Signal Processing, 1992. ICASSP-92., 1992 IEEE International Conference on], 4, $673-676$ vol.4 (Mar. 1992).

[5] Hampson, F. J. and Pesquet, J. C., "A nonlinear subband decomposition with perfect reconstruction," in [Acoustics, Speech, and Signal Processing, 1996. ICASSP-96. Conference Proceedings., 1996 IEEE International Conference on], 3, $1523-1526$ vol. 3 (May 1996).

[6] Gerek, O. N. and Cetin, A. E., "Adaptive polyphase subband decomposition structures for image compression," IEEE Transactions on Image Processing 9, 1649 -1660 (Oct. 2000).

[7] Gerek, O. N. and Cetin, A. E., "A 2-d orientation-adaptive prediction filter in lifting structures for image coding," IEEE Transactions on Image Processing 15, 106 -111 (January 2006).

[8] Le Pennec, E. and Mallat, S., "Image compression with geometrical wavelets," in [Image Processing, 2000. Proceedings. 2000 International Conference on], 1, 661 -664 vol.1 (2000).

[9] Claypoole, R. L., Davis, G. M., Sweldens, W., and Baraniuk, R. G., "Nonlinear wavelet transforms for image coding via lifting," Image Processing, IEEE Transactions on 12, 1449 - 1459 (December 2003).

[10] Kose, K. and Cetin, A. E., "Compressive sensing using the entropy functional," arXiv:1101.5079v2 [cs.IT] (Jan 2011).

[11] Pau, G., Pesquet-Popescu, A., and Piella, G., "Modified m-band synthesis filter bank for fractional scalability of images," Signal Processing Letters, IEEE 13(6), 345 - 348 (2006).

[12] Ansari, R., Kim, C. W., and Dedovic, M., "Structure and design of two-channel filter banks derived from a triplet of halfband filters," Circuits and Systems II: Analog and Digital Signal Processing, IEEE Transactions on 46, $1487-1496$ (Dec. 1999).

[13] Heller, P., "Lagrange m-th band filters and the construction of smooth m-band wavelets," in [TimeFrequency and Time-Scale Analysis, 1994., Proceedings of the IEEE-SP International Symposium on], 108 -111 (Oct. 1994).

[14] Pesquet-Popescu, B. and Bottreau, V., "Three-dimensional lifting schemes for motion compensated video compression," in [Acoustics, Speech, and Signal Processing, 2001. Proceedings. (ICASSP '01). 2001 IEEE International Conference on], 3, $1793-1796$ vol.3 (2001).

[15] Toreyin, B. U., Trocan, M., Pesquet-Popescu, B., and Cetin, A. E., "Lms based adaptive prediction for scalable video coding," in [Acoustics, Speech and Signal Processing, 2006. ICASSP 2006 Proceedings. 2006 IEEE International Conference on], 2, II (May 2006). 
[16] Smith, M. and Barnwell, T., I., "Exact reconstruction techniques for tree-structured subband coders," Acoustics, Speech and Signal Processing, IEEE Transactions on 34, 434- 441 (June 1986).

[17] Ansari, R., Guillemot, C., and Kaiser, J. F., "Wavelet construction using lagrange halfband filters," Circuits and Systems, IEEE Transactions on 38, 1116 -1118 (Sept. 1991).

[18] Piella, G., Pesquet-Popescu, B., and Heijmans, H., "Adaptive update lifting with a decision rule based on derivative filters," Signal Processing Letters, IEEE 9, 329 - 332 (Oct. 2002).

[19] Benazza-Benyahia, A., Pesquet, J.-C., and Krim, H., "A nonlinear diffusion-based three-band filter bank," Signal Processing Letters, IEEE 10, 360 - 363 (December 2003).

[20] Mallat, S., "Geometrical grouplets," APPLIED AND COMPUTATIONAL HARMONIC ANALYSIS 26, 161-180 (MAR 2009). 